\title{
CO-SEISMIC GRAVITY GRADIENT CHANGES OF THE 2006-2007 GREAT EARTHQUAKES IN THE CENTRAL KURIL ISLANDS FROM GRACE OBSERVATIONS
}

\author{
A. Rahimi ${ }^{\text {* }}$, M. Shahrisvand ${ }^{\mathrm{b}}$ \\ ${ }^{\text {a }}$ Geodesy division, Department of Surveying and Geomatic Engineering, College of Engineering, \\ University of Tehran, Iran-(armin_rahimi@ut.ac.ir) \\ ${ }^{\mathrm{b}}$ Remote sensing division, Department of Surveying and Geomatic Engineering, College of Engineering, \\ University of Tehran, Iran-(m.shahrisvand@ut.ac.ir)
}

KEY WORDS: GRACE observation, gravitational gradient changes, Kuril island earthquakes, PSGRN/PSCMP

\begin{abstract}
:
GRACE satellites (the Gravity Recovery And climate Experiment) are very useful sensors to extract gravity anomalies after earthquakes. In this study, we reveal co-seismic signals of the two combined earthquakes, the 2006 Mw8.3 thrust and 2007 Mw8.1 normal fault earthquakes of the central Kuril Islands from GRACE observations. We compute monthly full gravitational gradient tensor in the local north-east-down frame for Kuril Islands earthquakes without spatial averaging and de-striping filters. Some of gravitational gradient components (e.g. $\Delta \mathrm{Vxx}, \Delta \mathrm{Vxz}$ ) enhance high frequency components of the earth gravity field and reveal more details in spatial and temporal domain. Therefore, co-seismic activity can be better illustrated. For the first time, we show that the positive-negative-positive co-seismic $\Delta \mathrm{Vxx}$ due to the Kuril Islands earthquakes ranges from -0.13 to +0.11 milli Eötvös, and $\Delta \mathrm{Vxz}$ shows a positive-negative-positive pattern ranges from -0.16 to +0.13 milli Eötvös, agree well with seismic model predictions.
\end{abstract}

\footnotetext{
* Corresponding author
} 


\section{INTRUDUCTION}

A strong trust fault earthquake with magnitude $\mathrm{Mw}=8.3$ happened in the vicinity of the Central Kuril Islands, with a fault area as large as $250 \times 100 \mathrm{~km}^{2}$ and moment release between $1.6-5.3 \times 10^{21} \mathrm{~N} \mathrm{~m}$ (Ammon et al., 2008; Lay et al., 2009). It was strongest event of its type to be observed in the Pacific Ocean within the past 42 years since the catastrophic earthquake of March 28, 1964. On January 13, 2007, two months after the November 15, 2006 earthquake, another earthquake of similar magnitude $(\mathrm{Mw}=8.1)$ occurred in the region of the Central Kuril Islands with a fault area as large as $200 \times 40 \mathrm{~km}^{2}$ and moment release between $1.4-2.8 \times 10^{21} \mathrm{Nm}$ (Lay et al., 2009). The seismic parameters of the second earthquake differed significantly from those of the earlier earthquake and it was normal faulting events with bigger dip angle (Lay et al., 2009). Data from teleseismic networks, geodetic networks and tsunami sensors have been used to observe and model the co-seismic signature and slip history of 2006 trust event (Sladen et al, 2006; Ji et al 2006;Lay et al 2009) and 2007 normal event (Sladen et al, 2007; Ji et al 2007). In addition, space-borne gravimetry data from the Gravity Recovery And Climate Experiment (GRACE) satellites have been used to observe co-seismic and post-seismic signature of the 2006 and 2007Kuril Islands earthquakes (Han et al., 2016).

Large earthquakes lead to observable co-seismic and postseismic deformations, of which co-seismic deformation is a sudden and permanent jump, and post-seismic deformation consists of subsequent slip and slow recovery with time scale from months to years (or even longer) which is usually less than co-seismic changes. For example the 11 March $2011 \mathrm{Mw} 9.0$ Tohoku earthquake post-seismic slips (Ozawa et al., 2011) occurred with a moment of about $10 \%$ of the main shock. However, Kuril events is distinctly different; namely, the magnitude of post-seismic gravity change becomes by far larger than the co-seismic change within a few years (Han et al., 2016). Han et al (2016) also shows that the predicted total co-seismic gravity change of less than 1 micro-Gal at GRACE's spatial resolution. This was certainly below the detection threshold (Han et al., 2013).

Some Field quantities can better extract seismic signals other than geoid or gravity of the GRACE time-variable gravitational field. These other GRACE Field quantities include gravity gradients, vertical deflections, and gravitational gradient tensors (e.g., Li and Shen 2011; Sun and Zhou 2012; Wang et al. 2012a; Li 2015; Die 2015), which are sensitive to small-scale signals.

In this study we calculated a predicted total co-seismic gravity change of less than 1 which is consistent with Han et al (2016). Moreover, we calculate gravity change by the Release-05 (RL05) Level-2 monthly gravitational field data products and we show that the gravity signal is below the noise level. Furthermore, we compute full gravitational gradient tensor and show that some of its components are dominant of noise and reveal co-seismic gravitational gradient changes.

\section{GRACE DATA ANALYSIS}

We used the Release-05 (RL05) Level-2 monthly gravitational field data products released by University of Texas Center for Space Research (UTCSR), which are consisted of fully normalized SH coefficients complete to degree and order 60 . The $\mathrm{C} 20$ coefficients are replaced by satellite laser ranging (SLR) estimates (Cheng and Tapley 2004).

We use the SH coefficients Cnm, Snm used to compute monthly full gravitational gradients tensor in a local frame ( $x$ axis is at north direction, the $y$-axis to the east, and the $\mathrm{z}$ axis downward.)

In order to preserving original signals as much as possible we did not apply any de-striping and spatial smoothing procedure. To suppress seasonal variations and isolate coseismic change, we compute the difference between two mean gravity field after and before the earthquake, which is known as stacking approach based on that from Chen et al. (2007).

This difference include post-seismic signals associated earthquake. To remove the mentioned effects in the GRACE data, we adopt at a $1^{\circ} \times 1^{\circ}$ grid a time-dependent function.

$y(t)=a+b\left(1-e^{-t / \tau}\right)$

Where $\mathrm{a}, \tau$ and $\mathrm{b}$ are constant term, the relaxation time and total post-seismic gravitational gradient change. Then the co-seismic gravitational gradient changes were extracted by computing the difference between two mean gravity fields before and after earthquake.

\section{MODEL-PREDICTED GRAVITY CHANGE}

In order to predict gravity changes comparable with GRACE results we need two corrections which are free-air correction and seawater correction $(\mathrm{Li}, 2014)$.

First, we calculate gravity changes and vertical deformation with the half-space layered PSGRN/PSCMP code (Wang et al.2006). The calculated gravity changes contain contributions of both mass redistribution and effect of position changes at the calculating points because of the surface deformation. GRACE could not observe the latter contribution. After implementing the free-air correction (e.g. $\beta \Delta \mathrm{h}(\mathrm{x} 1, \mathrm{x} 2)$, where $\beta=0.3086 * 10-5 / \mathrm{s} 2$ takes into account the free-air effect due to the surface vertical motion of $\Delta \mathrm{h}$ ), we obtain the gravity changes on space-fixed points, which are attributed to mass redistribution caused by the earthquake (Sun et al. 2009)

Second, the dislocation theory above is assumed dry earth, so the deformation that occurred in the ocean bottom is replaced by sea water, As the mass redistribution due to seafloor deformations is partly compensated by seawater, the correction can be obtained by a Bouguer layer reduction based on seawater density and predicted vertical 
displacements of the seafloor (e.g. $-2 \pi G \rho_{w} \Delta h(x 1, x 2)$ where $\mathrm{G}$ denotes the Newton's gravitational constant, $\rho_{\mathrm{w}}=$ $1.03 \mathrm{gcm}-3$ represents the sea water density and $\Delta \mathrm{h}(\mathrm{x} 1, \mathrm{x} 2)$ is the co-seismic vertical displacement) (e.g, $\mathrm{Li}$ and Shen 2011; Wang et al. 2012; Li and Shen 2015).

Gravity changes predicted by dislocation models have a spatial resolution of several hundreds of kilometers. Thus, we need certain processing steps to make the model predictions consistent with GRACE results. In order to do that, we use $350 \mathrm{~km}$ isotropic Gaussian smoothing. The most commonly spatial smoothing method used is Gaussian smoothing (Wahr et al. 1998).

The calculated gravity change is transformed to geopotential spherical harmonic coefficients up to degree 60 . Then, each component of gravity gradient change is computed at a regular grid on Earth's mean semi-major axis $(6378.1363 \mathrm{~km})$ from the geo-potential coefficients up to the maximum degree (60) commensurable with the respective GRACE data products. The decomposition of a function on the sphere into a series of spherical harmonic is done by weighted least-square method (Sneeuw, 1994). And the calculated spherical harmonic coefficients are used to obtain full gravitational gradient tensor.

The dislocation model computation requires two types of inputs. One is an earth model reflecting geophysical properties of the crust and mantle in the earthquake region, and the other is a slip model describing the fault slips caused by the earthquake.

To show the procedure for the model prediction, we calculate co-seismic gravity changes before and after corrections for the $2006 \mathrm{Mw} 8.3$ thrust earthquake of the central Kuril Islands, which are depicted in Figure 2. We use (Lay et al., 2009) slip model (Figure 1). In addition, we use five layered earth model extracted from CRUST2 (table $1)$.

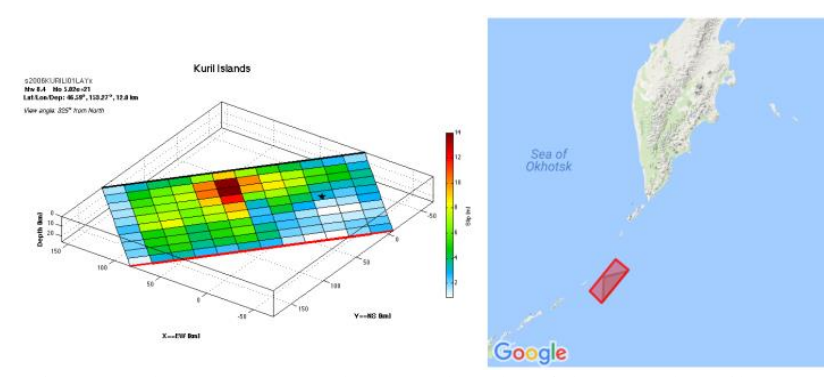

Figure 1. The 2006 Mw8.3 thrust earthquake of the central Kuril Islands fault slip distribution (Lay et al., 2009)
Table 1. The 5-layered half-space model of the 2006 Mw8.3 thrust earthquake of the central Kuril Islands (the parameters are from

\begin{tabular}{lllcccl}
\hline layer & Depth $(\mathrm{km})$ & $\mathrm{vp}[\mathrm{km} / \mathrm{s}]$ & $\mathrm{vs}[\mathrm{km} / \mathrm{s}]$ & $\rho\left[\mathrm{kg} / \mathrm{m}^{\wedge} 3\right]$ & $\begin{array}{l}\text { Viscosity } \\
\left(10^{18} \mathrm{~Pa}\right. \\
\mathrm{s})\end{array}$ & material \\
\hline 1 & 2 & 3.6 & 1.2 & 2200 & $\infty$ & $\begin{array}{l}\text { Elastic } \\
\text { body }\end{array}$ \\
2 & 6.00 & 5.6 & 3.34 & 2800 & $\infty$ & $\begin{array}{l}\text { Elastic } \\
\text { body }\end{array}$ \\
3 & 12.00 & 6.67 & 3.82 & 2900 & $\infty$ & $\begin{array}{l}\text { Elastic } \\
\text { body }\end{array}$ \\
4 & 22.00 & 7.44 & 4.06 & 3050 & $\infty$ & $\begin{array}{l}\text { Elastic } \\
\text { body }\end{array}$ \\
5 & 50.00 & 8.20 & 4.89 & 3400 & 1 & $\begin{array}{l}\text { Maxwell } \\
\text { body }\end{array}$ \\
\hline
\end{tabular}

The significant difference between figure $2 a$ and $2 b$ demonstrates the necessity of free-air correction. There could be evident difference between results with and without seawater correction, which can be clearly revealed also by the case of the $2006 \mathrm{Mw} 8.3$ thrust earthquake of the central Kuril Islands. Figure $2 \mathrm{c}$ represents the modelpredicted co-seismic gravity changes of the 2006 thrust earthquake after seawater correction for the oceanic areas. Compared with the prediction without seawater correction, the amplitude of the positive gravity changes in the near field decreases from 300 micro-Gal (figure 2b) to 150 micro-Gal (figure 2c). This illustrates that the compensation effect of seawater is non-negligible.

To make the model predictions consistent with GRACE results we need to smooth modeled-predicted gravity change. The smoothed results are depicted in figure $2 \mathrm{~d}$, from which we can find that the magnitude is reduced from several hundred of micro-Gal to several micro-Gal, and the spatial pattern is dominated by relatively larger scale features after $350 \mathrm{~km}$ Gaussian smoothing. 

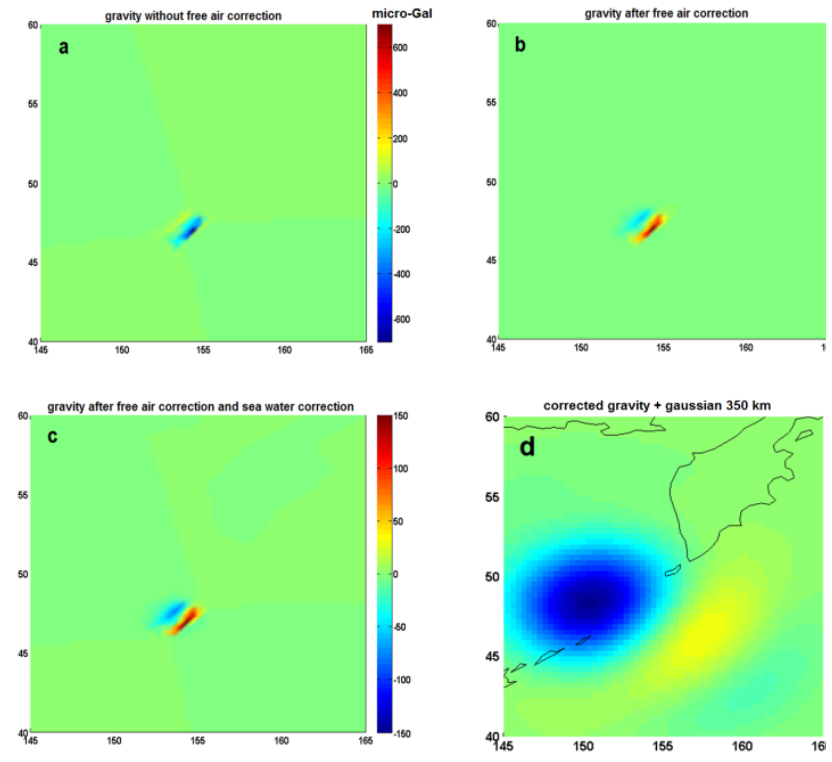

Figure 2. Dislocation model predictions of co-seismic changes due to the 2006 Mw8.3 thrust earthquake of the central Kuril Islands.

a) Gravity changes without free-air correction, b) Gravity changes after free-air correction , c) Gravity changes after free-air correction and seawater correction, and d) Corrected gravity changes smoothed with $350 \mathrm{~km}$ Gaussian filter. (Units in microGal)

\section{CO-SEISMIC SIGNAL OF KURIL ISLANDS EARTHQUAKES}

The 15 November 2006 Mw8.3 thrust and 13 January 2007 Mw8.1 normal fault earthquakes of the central Kuril Islands were strong enough to be detected by GRACE. Since these two earthquakes happened so close. We take the difference of two 2-year mean gravitational fields before and after the two combined earthquakes. The observation is a combination of co-seismic signals associated to the 2006 event and 2007 event, as well as the viscoelastic relaxation of the asthenosphere (Han et al, 2016). To remove the potential contamination from the post-seismic signals associated with these earthquakes the gravitational gradients of the months after the 13 January 2007 normal fault earthquake (from 2007 February to 2009 March) were used to fit a time-dependent function (equation 1).

Han et al (2016) found the gravity increase of $\sim 4$ microGals, observed consistently from various GRACE solutions around the epicentral area during 2007-2015, and it claimed that post-seismic gravity changes are greater than co-seismic changes. However, we show that co-seismic signals are big enough to be detected by GRACE by calculating $\Delta \mathrm{Vxx}, \Delta \mathrm{Vxz}$ components which are less polluted by noises. Furthermore, we show that gravity changes due to these combined earthquakes are below the noise level so some field quantities (e.g. $\Delta \mathrm{Vxx}, \Delta \mathrm{Vxz}$ ) can better extract seismic signals other than gravity of the GRACE time-variable gravitational field. And these two field quantity are sensitive to small-scale signals. We calculate predicted gravity changes of less than 1 micro-gal (figure 3d) and also we show GRACE-derived gravity changes (figure $3 \mathrm{c}$ ) which are below noise level.

For calculating predicted co-seismic gravity and gravity gradient changes, the finite fault models of the 2006 trust event (Sladen 2006), and the 2007 earthquakes (Sladen 2007), are used.

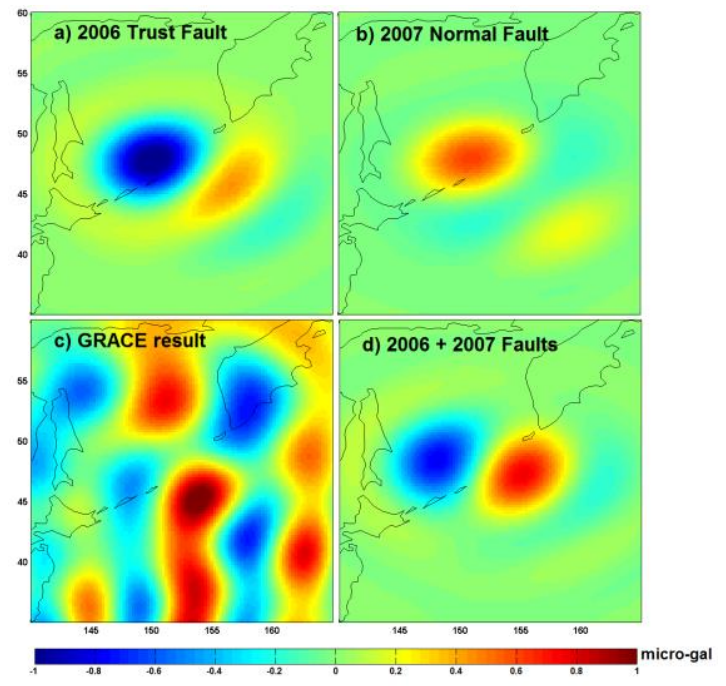

Figure 3. Co-seismic gravity changes a)Predicted gravity changes of the 2006 trust fault earthquake b)Predicted gravity changes of the 2007 normal fault earthquake c) GRACE result by taking the difference of two 2-year mean gravitational fields before and after these earthquakes d) Predicted gravity changes of both the 2006 and the 2007 Kuril Island earthquakes

Figure 4 represents gravitational gradient tensor of Kuril Islands earthquakes, first row is predicted gradient changes associated to the 2006 trust fault earthquake, second row is predicted gradient changes associated to the 2007 normal fault earthquake, third row is predicted gradient changes of both earthquakes, last row is GRACE-derived gradient changes. We know that GRACE stripe errors are distributed in north-south direction. Therefore by taking derivative to the $\mathrm{x}$-axis (north direction) these variations dramatically suppress. As you can see from figure $4, \Delta \mathrm{Vxx}$ and $\Delta \mathrm{Vxz}$ are dominant of noise level and show a maximum positive gradient changes of 0.11 milli Eötvös and 0.13 milli Eötvös respectively and the positivenegative-positive pattern of $\Delta \mathrm{Vxx}$ and $\Delta \mathrm{Vxz}$ distributed from north-west to south-east of Kuril Islands which agree well with model prediction. And other components polluted with other signal (e.g. striping and other noises). 


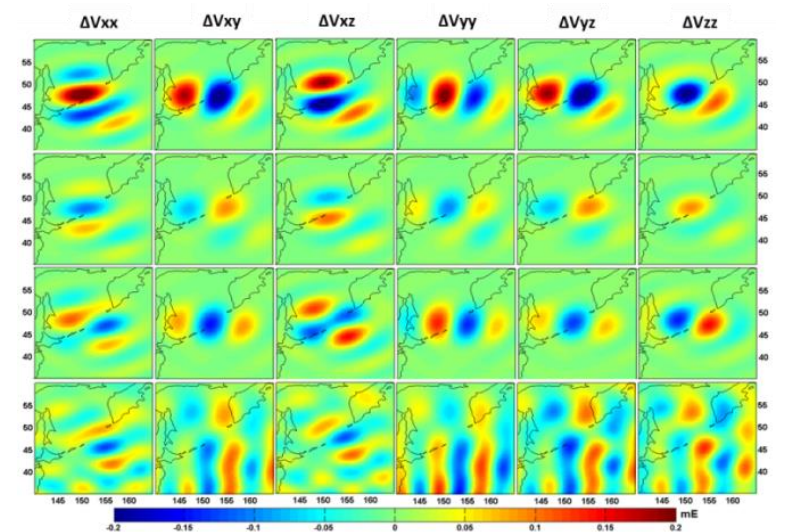

Figure 4. Modeled and observed gravitational gradient changes for Kuril island earthquakes. First row: Seismic model predicted total co-seismic gravitational gradient changes of the 2006 earthquake (trust fault), Slip model in use: (Sladen 2006), Second row: Seismic model predicted total co-seismic gravitational gradient changes of 2007 earthquake (normal fault), Slip model in use: (Sladen 2007),, Third row: Seismic model predicted total coseismic gravitational gradient changes of 2006+2007 earthquakes, Last row: GRACE-derived gravitational gradient changes, [units in milli Eötvös]

\section{CONCLUSION}

The 2006 and 2007 Kuril Islands earthquakes are detectable by GRACE. Monthly GRACE time variable gravity solutions are capable of monitoring some of gravitational gradient components (e.g. $\Delta \mathrm{Vxx}, \Delta \mathrm{Vxz}$ ) of two combined earthquakes. A positive-negative-positive pattern prevails in the spatial domain of the horizontal components of the gravity gradient changes due to the Kuril Islands earthquakes. And they agree well with model prediction. However, the predicted co-seismic gravity change computed less than 1 micro-gal which was below noise level. Therefore, the gravitational gradient components (e.g. $\Delta \mathrm{Vxx}, \Delta \mathrm{Vxz}$ ) are independent to GRACE stripy error and amplify high frequency components of gravity field which reveal more detail in spatial and temporal domain.

\section{REFRENCES}

Ammon, C. J., H. Kanamori, and T. Lay, "A great earthquake doublet and seismic stress transfer cycle in the central Kuril islands", Nature, 451(7178), 561-565, 2008.

Lay,T., H. Kanamori, C. J. Ammon, A. R. Hutko, K. Furlong, and L. Rivera "The 2006-2007 Kuril Islands great earthquake sequence", Jour. Geophys. Res., 114, B11308, doi:10.1029/2008JB006280, 2009

Sladen A. (Caltech, Kuril 2006). Preliminary Result 11/15/2006 “(Mw 8.3), Kuril islands, Source Models of Large Earthquakes". http://www.tectonics.caltech.edu/slip_history/2006_kuril/k uril.html, last accessed July 1, 2013.

C.Ji(UCSB,Kuril 2006).”Rupture process of the 2006 NOV 15 Magnitude 8.3 - KURIL Island Earthquake”

(Revised),http://earthquake.usgs.gov/earthquakes/eqinthene ws/2006/usvcam/finite_fault.php,last accessed September 5,2013 .

Sladen A. (Caltech, Kuril 2007). "Preliminary Result 01/13/2007 (Mw 8.1), Kuril islands, Source Models of Large Earthquakes".

http://www.tectonics.caltech.edu/slip_history/2007_kuril/k uril.html, last accessed July 1, 2013.

C.Ji(UCSB,Kuril 2007)."Rupture process of the 2007 Jan 13 Magnitude 8.1 - KURIL Island Earthquake" (Revised),http://www.geol.ucsb.edu/faculty/ji/big_earthqua kes/2007/01/13/kuril.html,last accessed August 22, 2013.

Han SC, J. Sauber and F. Pollitz. "Postseismic gravity change after the 2006-2007 great earthquake doublet and constraints on the asthenosphere structure in the central Kuril Islands”. Volume 43, Issue 7. Pages 3169-3177 DOI: 10.1002/2016GL068167, 2016.

Ozawa, S., T. Nishimura, H. Suito, T. Kobayashi, M. Tobita, and T. Imakiire, "Coseismic and postseismic slip of the 2011 magnitude-9 Tohoku-Oki earthquake", Nature, 475, 373-376, doi:10.1038/nature10227. 2011,

Li J, Shen WB “ Investigation of the co-seismic gravity field variations caused by the 2004 umatra-Andaman earthquake using monthly GRACE data”. J Earth Sci 22:280-291, doi:10. 1007/s12583-011-0181-x, 2011.

Zhou X, Sun W, Fu G “ Gravity satellite GRACE detects coseismic gravity changes caused by 2010 Mw8.8 Chile earthquake." Chin J Geophys 54(7):1745-1749. doi:10.3969/j. issn.0001-5733.2011.07.007, 2011.

Wang, L., Shum, C. K., Jekeli, C. "Gravitational gradient changes following the 2004 December 26 SumatraAndaman Earthquake inferred from GRACE”. Geophysical Journal International, 191(3), 1109-1118. doi: 10.1111/j.1365-246X.2012.05674.x, 2012. 
Li, Jin Wen-Bin Shen. "Monthly GRACE detection of coseismic gravity change associated with 2011 Tohoku-Oki earthquake using northern gradient approach." Earth, planets and Space. DOI 10.1186/s40623-015-0188-0, 2015.

Dai,C.,Shum,C.K.,Wang,R.,Wang,L.,Guo,J.,Shang,K.,Tapl ey,B "Improved constraints on seismic source parameters of the 2011 Tohoku earthquake from GRACE gravity and gravity gradient changes.Geophys".Res.Lett.41,1929-1936. http://dx.doi.org/10.1002/2013GL059178, 2014.

Cheng, M., Tapley, B. D. "Variations in the Earth's oblateness during the past 28 years", Journal of Geophysical Research, 109, B09402, doi:10.1029/2004JB003028. 2004.

Chen, J. L., Wilson, C. R., Tapley, B. D., Grand, S. GRACE "detects co-seismic and postseismic deformation from the Sumatra-Andaman earthquake". Geophysical Research Letters, 34, L13302. doi: 10.1029/2007g1030356. 2007.

Li J, Chen JL, Zhang ZZ. "Seismologic applications of GRACE time-variable gravity measurements". Earthquake Sci. 27(2):229-245, doi:10.1007/s11589-014-0072-1, 2014. Wang, R., F. Lorenzo-Martín, F. Roth. "PSGRN/PSCMP a new code for calculating co- and post-seismic deformation, geoid and gravity changes based on the viscoelastic-gravitational dislocation theory", Computers \& Geosciences, 32, 527-541, doi:

10.1016/j.cageo.2005.08.006. 2006.

Sun W, Okubo S, Fu G, Araya A. "General formulations of global co-seismic deformations caused by an arbitrary dislocation in a spherically symmetric earth model: applicable to deformed earth surface and space-fixed point”. Geophys J Int 177:817-833. doi:10.1111/j.1365246X.2009.04113.x, 2009.

Wahr, J., Molenaar, M., \& Bryan, F. "Time variability of the Earth's gravity field: Hydrological and oceanic effects and their possible detection using GRACE". Journal of Geophysical Research, 103(B12), 30205-30230,30229, 1998.
Sneeuw. "Global spherical harmonic analysis by leastsquares and numerical quadrature methods in historical perspective”. Geophys. J. Int., 1994 